\title{
Effects of Ion Optics on the Sensitivity Factors in Secondary Neutral Mass Spectrometry Measurements
}

\author{
Yoshikazu KIKUTA* and Holger JENETT** \\ *Analysis and Physical Properties Center, Showa Denko K. K., \\ 2-24-25, Tamagawa, Ohta, Tokyo 146, Japan \\ **Institut für Spektrochemie und Angewandte Spektroskopie (ISAS), \\ Bunsen-Kirchoff-Str. 11, D-4600 Dortmund 1, Germany
}

\begin{abstract}
The effects of the ion optics of the SNMS (secondary neutral mass spectrometry) INA-3 system on the intensity ratios, from which the relative sensitivity factors can be calculated, have been investigated for several III-V semiconductors: $\mathrm{GaAs}, \mathrm{GaP}$, and InP. It was found that the intensity ratios change significantly, depending on the electrode potentials of the ion optics and on the mass difference $(\Delta M)$ between the major constituent elements: within $90 \%$ regions of optimum electrode voltages and between $\Delta M=6$ and $84 \mathrm{amu}$, the maximum total relative deviation of relative intensities increases from about $10 \%$ to $250 \%$.
\end{abstract}

Keywords Secondary neutral mass spectrometry, ion optics, relative sensitivity factor

Because of its high sensitivity, mass spectrometry is widely applied to analyze solid inorganic materials. The dependency of different atomization and ionization methods has led to different kinds of mass analysis, such as secondary ion, glow discharge, and secondary (or sputtered) neutral mass spectrometry (SIMS, GDMS, SNMS). SNMS has attracted a lot of attention ${ }^{1-11}$ because of its advantages regarding quantitation and high depth resolution. The principles of plasma-SNMS and instrumental realization (Leybold INA-3) are described elsewhere. ${ }^{3-5,12}$

In principle, quantitation in plasma-SNMS is straightforward, since atomization (sputtering) and excitation (ionization) are decoupled, leading to a simple equation,

$$
\frac{C_{\mathrm{x}}}{C_{\mathrm{ref}}}=\frac{I_{\mathrm{x}} D_{\mathrm{ref}}}{I_{\mathrm{ref}} D_{\mathrm{x}}} .
$$

Here, $C, I$ and $D$ are the concentrations, intensities and sensitivity factors, respectively, of examples $\mathrm{x}$ and ref. ${ }^{5}$ The latter is usually taken as a matrix or common reference element $\left(e . g\right.$. Fe); the ratio $D_{\text {ref }} / D_{\mathrm{x}}$ is thus frequently written as the relative elemental sensitivity factor $\left(D_{x, \text { ref }}\right)$, for which empirical data have been compiled. ${ }^{3,13} D_{\mathrm{x}}$ can be written as

$$
D_{\mathrm{x}}=\gamma_{\mathrm{x}} \alpha_{\mathrm{x}}
$$

where $\gamma_{\mathrm{x}}$ is a product of the fraction of the flux of a sputtered particle $(x)$ which is ejected into the ion optics; the spectrometer transmission function for $\mathrm{x}$ and $\alpha_{\mathrm{x}}$ is the postionization probability. In a recent interlaboratory comparison of analysis results for several elements in an Al matrix ${ }^{15}$, a wide scatter of $D_{\mathrm{x}, \mathrm{Al}}$ was found. We have investigated the effects of INA-3 ion optics on the relative intensities, and found that a wide scatter of $D$ can be explained by the effects of the ion optics settings.

\section{Experimental}

\section{Samples}

Three III-V type semiconductors, GaAs, GaP and InP, (supplied by Showa Denko K.K., Tokyo) were measured, since the mass differences between the constituent elements cover a wide range between 6 (GaAs) and 84 (InP). Two measurement series were performed within $12 \mathrm{~d}$.

\section{Measurement conditions}

The interior of the plasma chamber in our Leybold INA-3 system had been changed to that described in ref. 16: the original stainless-steel surfaces had been covered or replaced by glass and Ta. Measurements were started after more than one hour from plasma ignition and sample introduction, when the plasma conditions had been stabilized. The $\mathrm{Cu}$ block around the specimen was cooled with liq. $\mathrm{N}_{2}$.

The bombardment voltage $\left(U_{\mathrm{DB}}\right)$ was set to $250 \mathrm{~V}$. The plasma features were an Ar pressure of $3.0 \times 10^{-3}$ mbar, a coil current of $5.5 \mathrm{~A}$, and an RF power of $200 \mathrm{~W}$. The Langmuir probes had a current of $325 \pm 5 \mu \mathrm{A}$ and a potential of $-2.1 \pm 0.1 \mathrm{~V}$. The quadrupole was operated with a resolution 7 ; using a $\mathrm{Cu} / \mathrm{Al}$ alloy containing 
$0.08 \mathrm{wt} \% \mathrm{~Pb}$, the quadrupole was tuned to the optimum peak resolution as well as sufficient sensitivity for the $\mathrm{Pb}$ isotopes. The multiplier voltages were set to $-2.7 \mathrm{kV}$ (conversion) and $2.9 \mathrm{kV}$.

\section{Ion optics}

For simplicity, the voltages on the electrodes of the ion optic system depicted in Fig. 1 are symbolized by the electrode symbols, themselves. According to ref. 17, L1, L2 and L3 form the extraction lens; LA together with a 90-degree deflector (ID and OD) the energy filter; and L7 and L8 the transfer lens to the quadrupole system, for which additionally the field axis potential FA can be defined.

The following optimization procedure was then performed: using the InP specimen, an attempt was made to obtain the optimum peak form maximum ${ }^{31} \mathrm{P}$ and ${ }^{115} \mathrm{In}$ and minimum ${ }^{40} \mathrm{Ar}$ intensities, first by varying of $\mathrm{FA}$, and then L1, L7, L8, OD, L4, L2 and L3. Following the INA-3 manual, ID was kept constant at $-300 \mathrm{~V}$. If it was not possible to obtain an identical potential for the maximum intensities of both analytical signals, an average value was chosen. Employing the settings obtained so far, the variation sequence was repeated, generally four times, until the last two steps of this iteration yielded identical settings. Using the starting set, it was ascertained that the optics setting was also

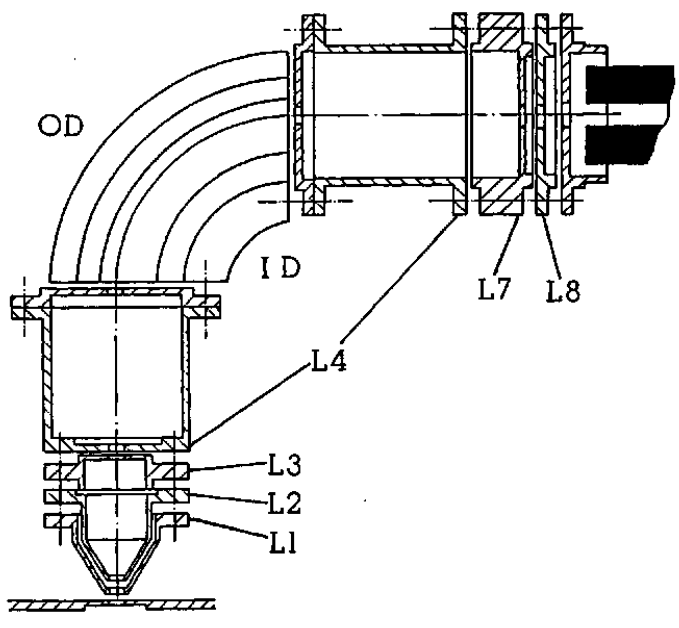

Fig. 1 INA-3 ion optics system (from ref. 17). applicable to the GaAs and GaP samples. The resulting set is shown in Table 1. In order to estimate the dependence of the relative intensities on the variation of the optics potentials, each electrode voltage, except for ID, was varied while keeping all others fixed at the values given in Table 1. The corresponding ${ }^{31} \mathrm{P},{ }^{69} \mathrm{Ga},{ }^{75} \mathrm{As}$ and ${ }^{115}$ In intensities, as issued by the data acquisition unit (DAU) in the cursor mode, were noted.

\section{Results and Discussion}

In Figs. 2 to 4, typical examples of the variation of the peak intensities with the lens voltages are shown for three specimens. The dotted lines are the optimized voltages listed in Table 1. The rather low intensities of the analytical signals (about 5 to $10 \times 10^{3} \mathrm{cps}$ ) are due to the restricted sample conductivities, low bombarding voltage, prolonged $(4 \mathrm{~mm})$ distance between the specimen and the plasma aperture, and a consequent suppression of any background; they are sufficiently

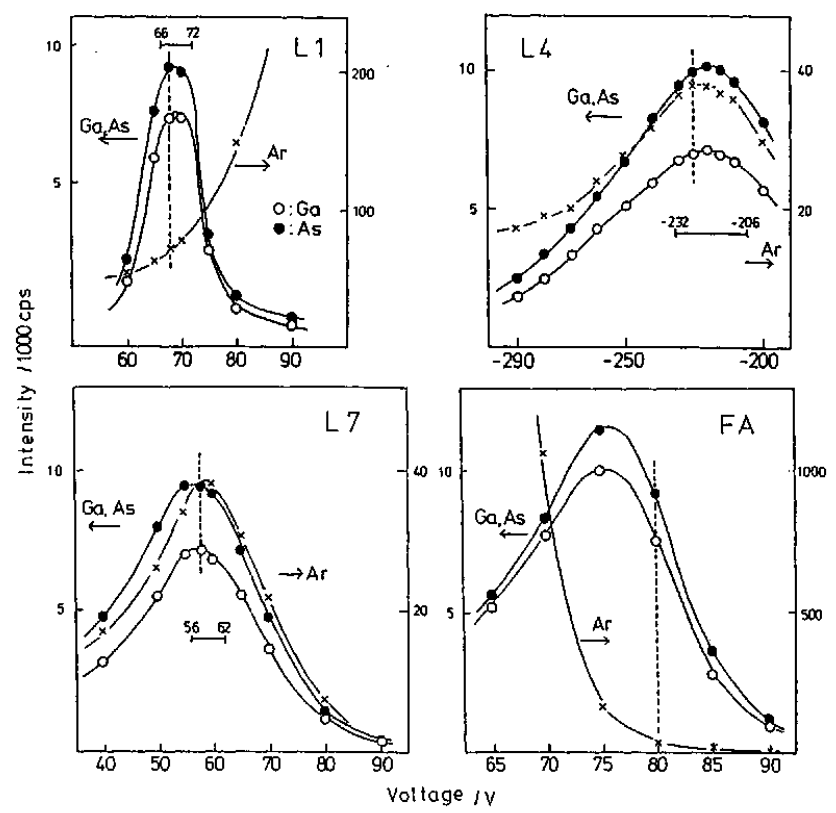

Fig. 2 GaAs: variation of intensities with electrode voltages. The dashed lines indicate the fixed voltages; the bars are the $90 \%$ ranges of Table 1 .

Table 1 Adopted set voltages and $90 \%$ ranges

\begin{tabular}{|c|c|c|c|c|c|c|c|c|}
\hline & $\mathrm{Ll}$ & $\mathrm{L} 2$ & L3 & L4 & OD & L7 & L8 & FA \\
\hline & \multicolumn{8}{|c|}{ Set voltage/eV } \\
\hline & 68 & 50 & -70 & -225 & -40 & 58 & -5 & 80 \\
\hline & \multicolumn{8}{|c|}{$90 \%$ range $/ \mathrm{eV}$} \\
\hline GaAs & $66-73$ & $33-57$ & $-120--37$ & $-232--206$ & $-42--39$ & $56-62$ & $-25-20$ & $78-82$ \\
\hline $\mathrm{GaP}$ & $66-70$ & $35-55$ & $-85--40$ & $-235--215$ & $-42--39$ & $55-62$ & $-20-20$ & $78-82$ \\
\hline InP & $66-69$ & $26-50$ & $-120--44$ & $-232--216$ & $-41--38$ & $55-60$ & $0-20$ & $78-82$ \\
\hline
\end{tabular}




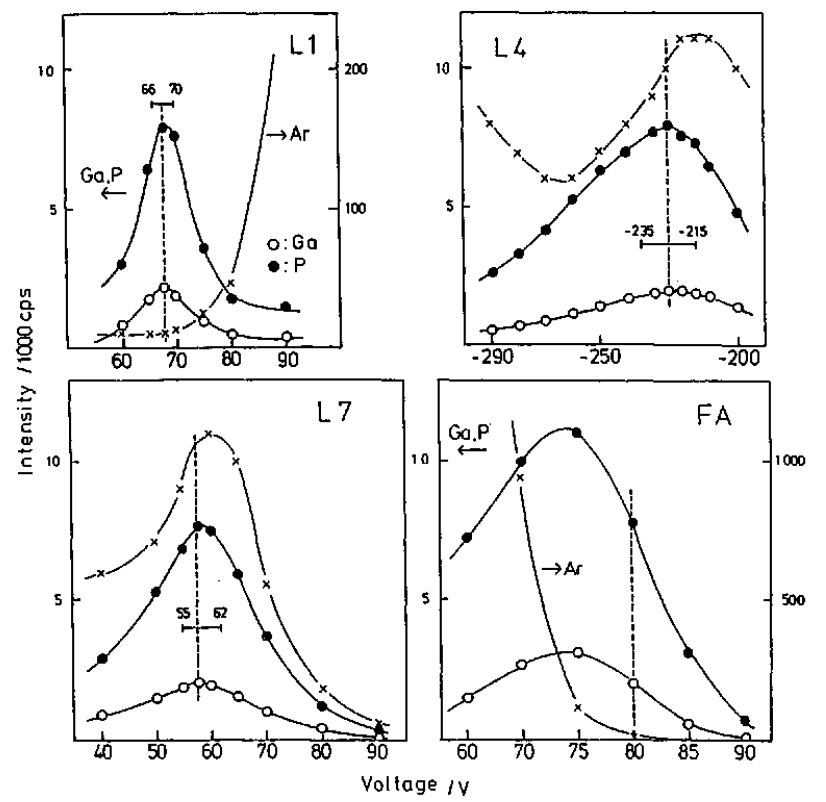

Fig. 3 GaP, see Fig. 2.

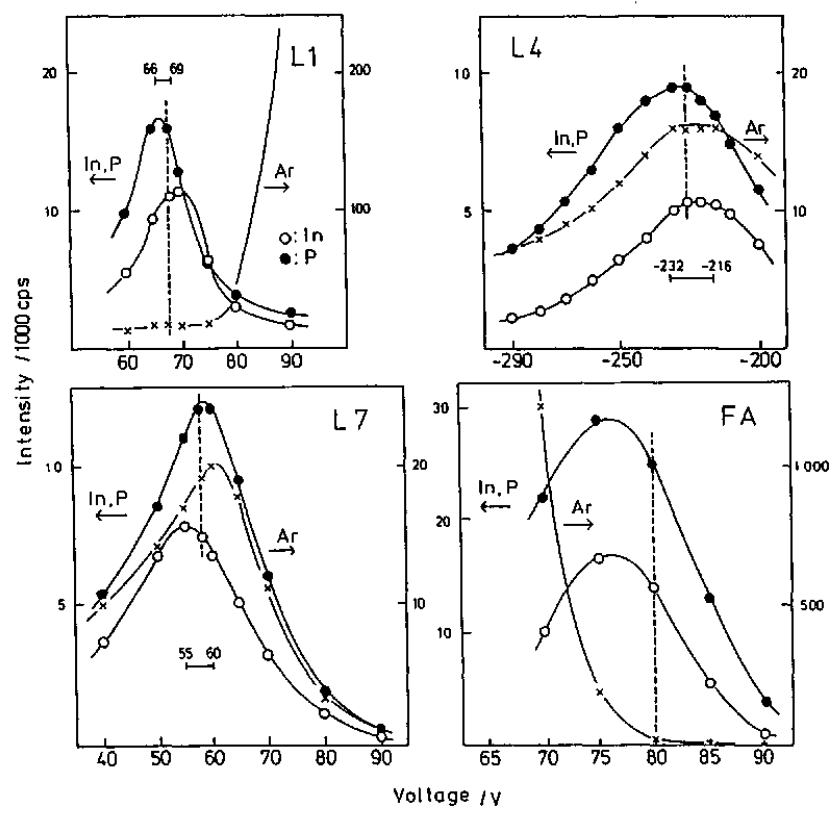

Fig. 4 InP, see Fig. 2.

large to keep any statistical errors small. The differing absolute intensities among the samples are partly due to the different conductivities.

From FA and L1, it can be seen that residual gas discrimination is effectively accomplished. Since FA acts as a part of the discriminator system for thermal ions when it exceed the plasma potential by more than $8 \mathrm{~V}^{17}$, the latter can roughly be estimated to lie between 60 and $70 \mathrm{~V}$ under the given conditions.

In the case of GaAs, for which the mass difference between the constituents is small, the maximum

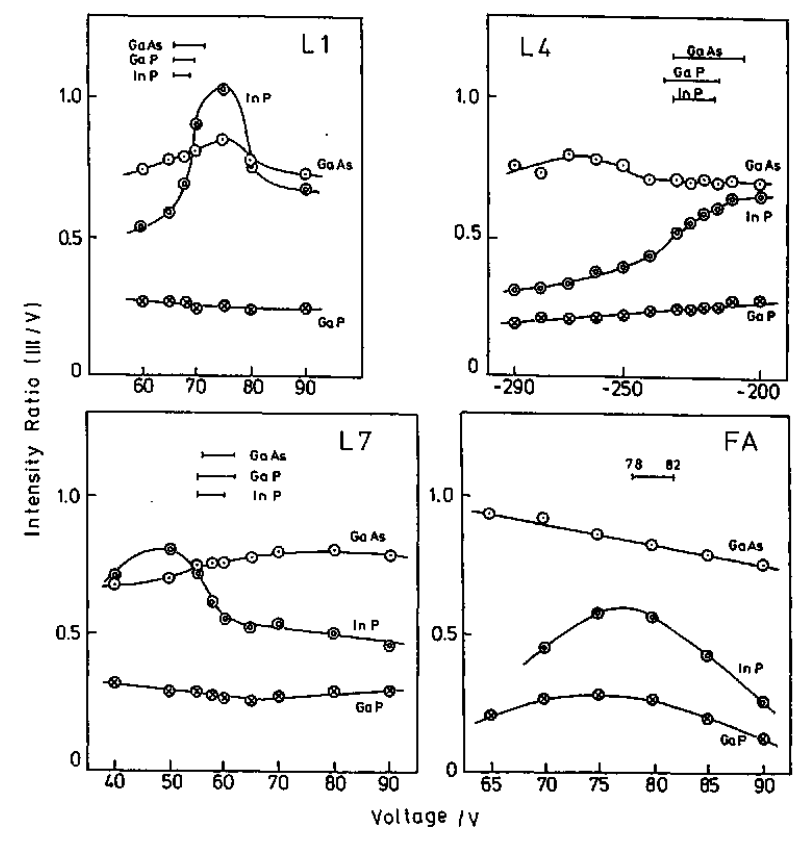

Fig. 5 Variation of the intensity ratios (see Fig. 2).

intensities are transmitted at nearly identical voltages; in InP, however, which has the greatest mass difference, the voltage shifts between the maximum intensities of In and $P$ can be observed. These shifts - as well as the smaller ones for $\mathrm{GaP}$-increase with the mass difference of the sputtered particles.

Figure 5 shows the intensity ratios calculated from each point in Figs. 2 to 4 . The intensity of each groupIII element is divided by the corresponding group-V value. Again, the variations of the relative intensities of InP are greater than those of GaAs. In practice this means that if, e.g., $\mathrm{L} 1$ is tuned to maximum ${ }^{31} \mathrm{P}$ or ${ }^{115} \mathrm{In}$ intensity ( 66 or $70 \mathrm{~V}$, respectively), one obtains an $I_{\text {In }} / I_{P}$ ratio of 0.6 to 0.9 , respectively, which would already yield a $50 \%$ deviation in relative $D$. Similar calculations can be performed for the other electrodes.

In the following, we attempt to clarify to what extent variations of the ion optics might contribute to such relative $D$ variations. The voltage range in which more than $90 \%$ of the individual maximum intensities lie can be read for all cases. For instance, for L1 and GaAs it lies between 66 and $72 \mathrm{~V}$. The meaning of this arbitrary range is that it may represent an optimum electrode setting for one specific compound with possible slight aberrations. When the voltages for the maximum intensities of the two constituents are different, as is the case for InP, only range overlaps are adopted. In the case of FA, a narrow voltage range (78 to $82 \mathrm{~V}$ ) near the maximum intensity with an optimum peak shape and minimum $\mathrm{Ar}^{+}$intensity is selected. Every range is also listed in Table 1.

Even with these individual $90 \%$ (or optimum feature) ranges, the intensity ratios change significantly (see Fig. 5). As a possible criterion for the dependence of 
the relative sensitivity factors on electrode $i$ voltages, the highest relative intensities in the $90 \%$ range are divided by the lowest ones, thus yielding relative deviations $S_{\mathrm{i}} \geq 1$. For instance, in the case of L1 and InP, where the $90 \%$ range lies between 66 and $69 \mathrm{~V}$ (Fig. 4), the highest relative III/ $\mathrm{V}$ intensity $(0.77)$ is found at $69 \mathrm{~V}$ and the lowest one (0.61) at $66 \mathrm{~V}$ (Fig. 5). From this, $S_{\mathrm{LI}}=0.77$ / $0.61=1.26$. Together with the mass difference $(\Delta M)$, all $S_{\mathrm{i}}$ values of both measuring series are given in Table 2 , in which the upper values refer to Figs. 2 to 5.

All lenses show an $S_{\mathrm{i}}$ increase of about $20 \%$ for a $\Delta M$ of roughly 100 . If $S$ of each lens is independent of the others, and if the mistuning of each individual electrode is amplified by those of all others, the total deviations $\left(S_{\text {total }}\right)$ can be calculated according to

$$
S_{\mathrm{total}}=S_{\mathrm{L} 1} S_{\mathrm{L} 2} S_{\mathrm{L} 3} S_{\mathrm{L} 4} S_{\mathrm{OD}} S_{\mathrm{L} 7} S_{\mathrm{L} 8} S_{\mathrm{FA}}
$$

which are also listed in Table 2. The reproducibility of $S_{\text {total }}$ can be also found. Since $S_{\mathrm{i}} \geq 1$ in any case and $S_{\mathrm{i}}$ generally increase with $\Delta M$, one obtains an exponential increase of $S_{\text {total }}$ with $\Delta M$ (Fig. 6). And since all electrodes show a linear dependence of $S$ to the mass difference (where about $20 \%$ deviation is seen for mass

Table 2 Deviations of intensity ratios $S_{\mathrm{i}}$ for electrodes $\mathrm{i}$

\begin{tabular}{lcccccccccc}
\hline & $\Delta M$ & $S_{\mathrm{L} 1}$ & $S_{\mathrm{L} 2}$ & $S_{\mathrm{L} 3}$ & $S_{\mathrm{L} 4}$ & $S_{\mathrm{OD}}$ & $S_{\mathrm{L} 7}$ & $S_{\mathrm{L} 8}$ & $S_{\mathrm{FA}}$ & $S_{\text {total }}$ \\
\hline GaAs & 6 & 1.06 & 1.00 & 1.00 & 1.00 & 1.07 & 1.03 & 1.00 & 1.03 & 1.20 \\
& & 1.00 & 1.00 & 1.02 & 1.03 & 1.00 & 1.03 & 1.00 & 1.03 & 1.11 \\
$\mathrm{GaP}$ & 38 & 1.00 & 1.08 & 1.08 & 1.04 & 1.04 & 1.08 & 1.09 & 1.17 & 1.74 \\
& & 1.07 & 1.03 & 1.03 & 1.15 & 1.06 & 1.05 & 1.09 & 1.14 & 1.86 \\
$\mathrm{InP}$ & 84 & 1.26 & 1.09 & 1.18 & 1.22 & 1.06 & 1.27 & 1.17 & 1.18 & 3.67 \\
& & 1.23 & 1.11 & 1.07 & 1.20 & 1.06 & 1.26 & 1.13 & 1.17 & 3.10 \\
\hline
\end{tabular}

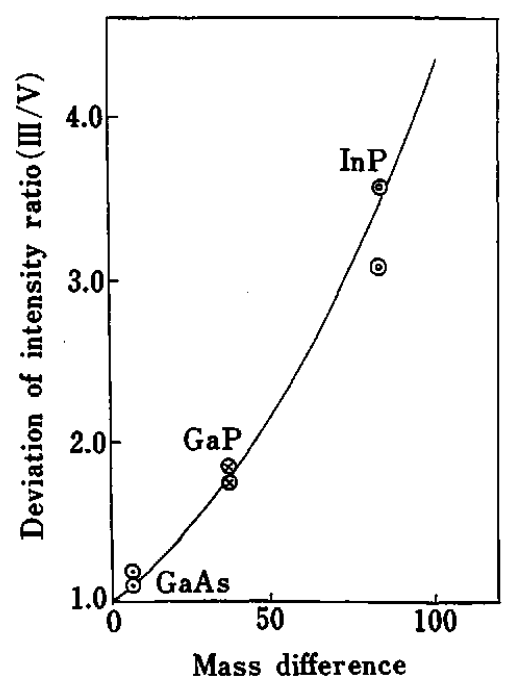

Fig. 6 Correlation between the deviation of the intensity ratios and the mass difference. The solid line shows $S_{\text {total }}=(0.002 \times \Delta M+1)^{8}$. difference 100$), S_{\text {total }}$ can be roughly expressed as

$$
S_{\text {total }}=(0.002 \times \Delta M+1)^{8}
$$

The difference between $S_{\text {total }}$ and unity (the latter would reflect the ideal case of mass independency) can be regarded as the possible range of the ion optics contribution to the experimental data.

Figure 6 suggests that the comparability of sensitivity factors ultimately requires a comparable adjustment of the ion optics using identical mass peaks. In our experiments, the intensity ratio of $I_{\mathrm{In}} / I_{\mathrm{P}}$ optimized with In was 1.24 , while with $P$ it was 0.45 ; the deviation between two optimization methods, using In or $\mathbf{P}$, is 2.75 . This value is less than the predicted deviation value of $S_{\text {total }}(3.10,3.67)$ shown in Table 2, because the maximum peak positions of In and $\mathrm{P}$ in each electrode (except for L1 and L8) are within $90 \%$ range, and partly because neither electrode is independent. However, the deviation is still large and phenomenally supports the idea that each electrode can be considered as being nearly independent at the others.

From the manual of this instrument ${ }^{17}$, the energy window and pass energy are described as

$$
\Delta E=-(\mathrm{ID}-\mathrm{OD})-\mathrm{const} \text {. }
$$

and

$$
E_{\text {pass }}=-(\mathrm{ID}-\mathrm{OD})+\mathrm{L} 4+\text { const. }
$$

respectively. When ID and OD are constant, the change in the L4 voltage shows the energy distributions of ions; for the L4 voltage shift between the maximum intensity of In and P in Fig. 4 it is supposed that the energy distributions of $\mathbf{P}$ (light element) and In (heavy element) are different. On the other hand, the energy distributions of $\mathrm{Ga}$ and As (between which mass difference is small) are thought to be identical, since the peak positions of the maximum intensities are nearly identical in Fig. 2. Since ion optics serve as an energy filter in this quadrupole mass spectrometer, it is suggested that the large deviation of $S_{\text {total }}$ is reflected by the different ion energy distributions, thus explaining the different masses between constituents.

Much care should be required when the relative sensitivity factors of a materials with a large mass (like InP) are quoted from other references, even though the instruments used are the same and the other conditions (for instance, the plasma condition and target potential) are also the same. It should be necessary that it be described in reports how the optimization of ion optics was achieved.

Recently interlaboratory round-robin measurements of several elements in Al alloy were carried out by 13 users of the INA-3 system in Germany; ${ }^{15}$ however the sensitivity factors of elements to $\mathrm{Al}, D_{\mathrm{x}, \mathrm{Al}}$, were very much scattered between laboratories, even though the target potential was the same, and the degree of deviation 


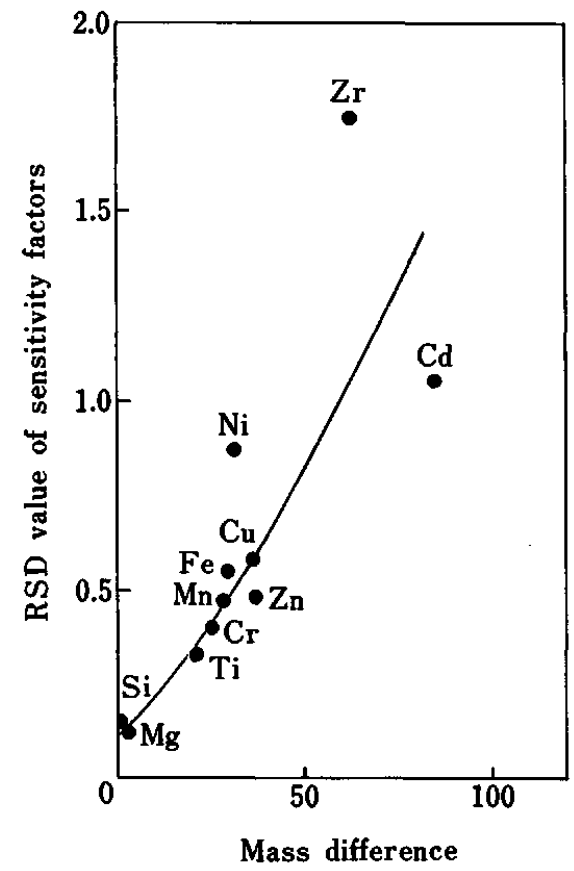

Fig. 7 Correlation between the RSD (relative standard deviation) value of the obtained sensitivity factors $\left(D_{\mathrm{x}, \mathrm{Al}}\right)$ in round-robin measurement and mass difference to $\mathrm{Al}$.

of the obtained sensitivity factors had a tendency for mass dependency. Figure 7 shows the correlation between the relative standard deviation (RSD value) of the obtained sensitivity factors and mass difference. A significantly large mass dependency can be found that is very similar to the effects of the ion optics shown in Fig. 6. We think that the wide scatter of sensitivity factors in Fig. 7 can be mostly explained by the different setting of the ion optics among these laboratories.

We have been investigating such effects of the electrostatic analyzer (ESA) in sector-type mass spectrometers, such as GDMS and SIMS; however, the deviations in the intensity ratio caused by ESA tuning are very small, within $10 \%$ even in the case of InP. On the other hand, the deviations of the intensity ratio and its mass dependency were found in a quadrupole-type ICP-MS (inductively coupled plasma mass spectrometer). It is thought that the large effects of the ion optics setting is found intrinsically in the quadrupole-type mass spectrometer which does not have an ion reflector or a sector type ESA. Details will be discussed later comparing the results of several kinds of mass spectrometers (in preparation).

\section{References}

1. W. Reuter, Tr. Anal. Chem., 8, 203 (1989).

2. P. K. Chu, J. C. Hunke and R. J. Blattner, J. Vac. Sci. Technol., A5, 295 (1987).

3. R. Jede, H. Peter, G. Dunnebier, O. Ganschow, U. Kaiser and K. Seifert, J. Vac. Sci. Technol., A6, 2271 (1988).

4. K. H. Muller and H. Oechsner, Mikrochim. Acta [Wien], 1983, Suppl. 10, 51.

5. H. Oechsner, Topics Current Phys., 37, 63 (1984).

6. T. Ishitani, N. Sakudo, H. Tamaru and I. Kanomata, Phys. Lett., 68A, 375.

7. K. F. Muller, K. Seifert and M. Wilmers, J. Vac. Sci. Technol., A3, 1367 (1985).

8. A. Wucher, J. Vac. Sci. Technol., A6, 2287 (1988).

9. E. Stumpe, H. Oechsner and H. Schoof, Appl. Phys., 20, 55 (1979).

10. H. Oechsner H. Paulus and P. Bachmann, J. Vac. Sci. Technol., A3, 1403 (1985).

11. R. Jede, U. Kaiser, H. Peter, O. Ganschow and J. P. Denis, "Secondary Ion Mass Spectroscopy SIMS VT", "Proc. 6th Int. Conf. Versailles 1987", ed. A. Benninghoven, A. M. Huber and H. W. Werner, p. 353, Wiley, New York.

12. H. Oechsner and E. Stumpe, Appl. Phys., 14, 43 (1977).

13. A. Wucher F. Novak and W. Reuter, J. Vac. Sci. Technol., A6, 2265 (1988).

14. A. Wucher and W. Reuter, J. Vac. Sci. Technol., A6, 2316 (1998).

15. J. Bartella et al., to be published.

16. A. Wucher, J. Vac. Sci. Technol., A6, 2293 (1988).

17. INA-3 Ion optics manual, Leybold AG, Cologne, FRG.

(Received July 12, 1991)

(Accepted August 12, 1991) 